\title{
Postoperative analgesia after minimally invasive thoracoscopy: What should we do?
}

\author{
Steven M. Neustein, MD • Patrick J. McCormick, MD
}

Received: 22 December 2010/Accepted: 14 February 2011/Published online: 24 February 2011

(c) Canadian Anesthesiologists' Society 2011

Many of the thoracic operations that previously required a thoracotomy incision can now be performed with several small incisions and video-assisted thoracic surgery (VATS). Although there have been reports of less pain and less impairment of pulmonary function with VATS compared with thoracotomy, ${ }^{1}$ many VATS patients report significant acute and chronic postoperative pain. Treating thoracoscopic pain effectively and minimizing the later development of chronic pain requires familiarity with a wide spectrum of analgesic techniques.

An important variable in the analgesic plan for VATS is the likelihood of converting to an open thoracotomy. Conversion may be due to technical difficulties, inadequate lung separation, or bleeding, and may be more likely when the surgeon has less experience with VATS. The likelihood of conversion to an open procedure must be assessed preoperatively. If a VATS procedure is converted to a thoracotomy and analgesia (such as an epidural) is not in place, the patient's recovery may be impaired. If not wellcontrolled, postoperative thoracic pain can lead to splinting and may impair the patient's ability to take deep breaths following thoracic surgery.

It is difficult to state precisely the incidence of pain following thoracoscopy, as the extent and severity varies according to surgical technique, patient sensitivity, and anesthetic pain management. In a recent survey of Dutch thoracic surgery patients, $47 \%$ of the VATS patients reported chronic pain. ${ }^{2}$ In an earlier study, the incidence of chronic pain was reported to be $36 \%$ in VATS patients

S. M. Neustein, MD (ه) · P. J. McCormick, MD Department of Anesthesiology, Mount Sinai Medical Center, The Mount Sinai School of Medicine, One Gustave L. Levy Place, New York, NY 10029-6574, USA

e-mail: steve.neustein@mountsinai.org undergoing wedge resection. ${ }^{3}$ It might seem intuitive that performing the surgery by thoracoscopy, which utilizes small incisions, would lead to less postoperative pain. However, the introduction of trocars during thoracoscopy may injure intercostal nerves, and additional manipulation of these instruments during the surgery may traumatize these nerves further by compression against ribs. ${ }^{4}$ If a lobectomy is being performed, an incision will need to be enlarged in order to extract the specimen, and a rib retractor may be required. Also, the duration of the surgery may be greater using VATS. Ipsilateral shoulder pain is a common complaint following VATS, and in one study, it was reported to be chronic in $10 \%$ of patients. ${ }^{5}$

Acute postoperative pain may be due to myofascial or neuropathic processes producing neuroplastic changes that lead to chronic pain. Peripheral and central sensitization can occur. The goal of preemptive analgesia is to prevent these neuroplastic changes from occurring and avoiding what is commonly referred to as a spinal windup. The conclusion of a meta-analysis of 66 studies of patients undergoing a variety of different types of surgery was that epidural analgesia, wound infiltration, and systemic nonsteroidal anti-inflammatory drug (NSAID) administration are effective preemptive analgesic techniques, but systemic opioids are not. ${ }^{6}$ While the theory is enticing, there are no studies examining the efficacy of preemptive analgesia on thoracoscopic procedure pain. Evidence of efficacy is required, particularly for chronic postoperative thoracoscopic pain, before more practitioners administer preemptive analgesia.

An epidural placed at the $\mathrm{T} 4$ level provides highly effective analgesia for incisional pain following thoracotomy. However, the data showing that epidural analgesia improves pain control following thoracoscopy are not uniformly positive. Furthermore, patients taking 
anticoagulation or antiplatelet therapy may not be eligible for epidural analgesia. In a randomized trial in VATS patients, it was found that patients with epidural analgesia had more effective pain control through the first postoperative day $v s$ control patients without an epidural. ${ }^{7}$ The control group patients required additional analgesics at higher total doses than the epidural group. However, in a prior study, thoracic epidural analgesia did not reduce the incidence of either acute or chronic pain following VATS pleurectomy for primary spontaneous pneumothorax. ${ }^{8}$ Single-shot intrathecal morphine is not commonly used for VATS patients, and to our knowledge, there are no studies discussing this modality in VATS.

Placement of a paravertebral block (PVB) is a popular alternative to epidural analgesia. In a recent survey of thoracic anesthesiologists in the United Kingdom, it was found that the use of PVB with a morphine patient-controlled analgesia (PCA) for VATS lobectomy was much more common $(50 \%)$ than thoracic epidural analgesia $(10 \%){ }^{9}$ An injection of local anesthetic at the T4 paravertebral level can produce ipsilateral blockade from the T2 to T6 dermatomes. Unlike an epidural anesthetic, a PVB does not cause a sympathectomy, thus hypotension is less frequent. A PVB can be accomplished with either a single injection or the placement of a catheter. There is a quantity of encouraging data regarding the use of PVB for VATS. In 2005, Vogt et al. demonstrated that PVB improved postoperative pain in thoracoscopy patients up to $48 \mathrm{hr}$ after surgery. ${ }^{10}$ In two subsequent trials, multilevel PVB was found to reduce pain scores and coughing, but only in the first four hours and six hours postoperatively, respectively. ${ }^{11,12}$ Due to the efficacy of PVB, an epidural is not necessary for VATS procedures with a low probability of conversion to thoracotomy.

Intercostal blocks may also be performed and can be done after the patient has been positioned in the lateral position for the surgery and while under general anesthesia. The risk of a pneumothorax following intercostal block is minimized when done for thoracic surgery, as the operative lung is to be collapsed during the surgery, and a chest tube will be placed regardless. This technique may also be considered if there is a contraindication to neuraxial block, such as in coagulopathy. In relatively minor VATS, such as drainage of pleural fluid and biopsy, the procedure can be done utilizing intercostal blocks and intravenous sedation only. In those cases, the lung ipsilateral to the surgery will collapse during spontaneous respiration, and a specific anesthetic technique to provide lung isolation will not be needed. The intercostal nerve blocks can provide both intraoperative anesthesia and postoperative analgesia. The use of intercostal nerve blocks has been reported to reduce both pain and narcotic requirements postoperatively following VATS. ${ }^{13}$ Bupivacaine administered via the chest tube has also been reported to reduce postoperative pain scores and opioid usage following VATS. ${ }^{14}$

Multimodal analgesia reduces opioid requirements and side effects, including respiratory depression, nausea, and pruritis. Patients undergoing thoracic surgery usually have a history of cigarette smoking, may have chronic obstructive pulmonary disease, and may not tolerate the respiratory depressant effects of systemic opioids. While many anesthesiologists, including ourselves, regularly use NSAIDs postoperatively, there is minimal specific evidence for this practice in thoracoscopic surgery patients. In one report, a consecutive series of VATS patients were treated successfully for postoperative pain with up to two days of a subcutaneous morphine infusion and regular NSAID administration. ${ }^{15}$ Some surgeons may prefer to delay administration of ketorolac until the post-operative period due to concern for potential inhibitory effects on the coagulation system. Ketorolac may be helpful with shoulder pain, which is not likely to be ameliorated with epidural analgesia.

A powerful indication for specific postoperative analgesia techniques in VATS patients would be improved outcomes, but there is a scant amount of data on this topic. In a retrospective study, it was found that fewer patients developed a postoperative supraventricular tachycardia following thoracoscopy if an epidural had been placed previously. ${ }^{16}$ A subsequent prospective randomized study demonstrated reduced incidence of arrhythmias in patients with epidural analgesia following pulmonary resection in lung cancer patients. ${ }^{17}$ However, that study was not limited to thoracoscopy.

It is our view that the postoperative analgesia plan for a VATS patient begins with an assessment of the extent of the procedure, i.e., the likelihood of conversion to an open thoracotomy. Many short-duration VATS do not require a thoracic epidural and its attendant risks. A patient having this kind of limited procedure may require only intercostal blocks, ketorolac, and an opioid PCA for postoperative pain. An epidural would not be required. NSAIDs should also be used, barring contraindications.

A patient who is to have an extensive resection with a high chance of opening should have a thoracic epidural catheter placed and a PCA activated immediately after surgery. Some surgeons may convert routinely to a thoracotomy if the initial biopsy with VATS is positive for cancer, and an epidural should be placed routinely for those patients unless there is a contraindication.

It is our view that there are several research opportunities to discover the factors that determine the technique to be utilized for each VATS. In our practice, the decision to place a thoracic epidural in a VATS patient depends on the anesthesiologist's personal knowledge of the surgeon, the procedure, and the patient. Observational and 
prospective studies of the factors involved in the analgesic plan and the outcomes would be extremely helpful.

Beyond the recovery room, measuring outcomes after VATS and linking them to the perioperative analgesic plan is a necessary step in research. Everything from a patient's satisfaction to the development of chronic pain to postoperative complications may be linked to the analgesic plan, but we have no data to support or refute this idea. Current research validates that modern postoperative analgesic techniques are improving the recovery of VATS patients, but more in-depth analysis is required.

\section{L'analgésie postopératoire après une thoracoscopie mini-invasive: que devrions-nous faire?}

Nombre d'interventions thoraciques qui nécessitaient une thoracotomie par le passé peuvent aujourd'hui être réalisées à l'aide de plusieurs petites incisions et par vidéothoracoscopie. Bien que certains comptes rendus rapportent une douleur moindre et moins de dysfonction pulmonaire avec la vidéothoracoscopie qu'avec la thoracotomie, ${ }^{1}$ plusieurs patients ayant subi une vidéothoracoscopie rapportent néanmoins d'importantes douleurs postopératoires aiguës et chroniques. Le traitement efficace de la douleur thoracoscopique et la réduction de la manifestation subséquente de douleur chronique nécessitent la connaissance d'une vaste gamme de techniques analgésiques.

Une variable importante dans le plan d'analgésie lors d'une vidéothoracoscopie est la probabilité de devoir réaliser une thoracotomie ouverte. La conversion de l'approche chirurgicale pourrait être due à des difficultés techniques, à un isolement pulmonaire inadapté ou au saignement, et pourrait être plus probable si le chirurgien ne possède que peu d'expérience en vidéothoracoscopie. La probabilité de conversion vers une intervention ouverte doit être évaluée avant l'opération. Si une intervention vidéothoracoscopique se change en thoracotomie et qu'une analgésie (par ex. une péridurale) n'est pas installée, la récupération du patient pourrait en souffrir. Mal contrôlée, la douleur thoracique postopératoire peut entraîner une hypoventilation réflexe et diminuer la capacité du patient à prendre de grandes inspirations après une chirurgie thoracique.

Il est difficile d'établir précisément l'incidence de douleur post-thoracoscopie, étant donné que l'étendue et la gravité varient selon la technique chirurgicale, la sensibilité du patient et la prise en charge de la douleur par l'anesthésiste. Dans un récent sondage portant sur des patients de chirurgie thoracique hollandais, $47 \%$ des patients ayant subi une vidéothoracoscopie ont rapporté de la douleur chronique. ${ }^{2}$ Dans une étude antérieure, l'incidence de douleur chronique était de $36 \%$ chez les patients ayant subi une vidéothoracoscopie et subissant une résection cunéiforme périphérique. ${ }^{3}$ Intuitivement, le fait de réaliser une chirurgie par thoracoscopie, en utilisant de petites incisions, devrait provoquer moins de douleur postopératoire. Toutefois, l'introduction de trocarts pendant la thoracoscopie pourrait léser les nerfs intercostaux, et une manipulation supplémentaire de ces instruments pendant la chirurgie pourrait traumatiser ces nerfs encore davantage en les comprimant contre les côtes. ${ }^{4}$ En cas de lobectomie, une incision devra être élargie afin d'extraire le spécimen, et un écarteur pourrait être nécessaire. De plus, la durée de la chirurgie peut être prolongée lorsqu'on réalise une vidéothoracoscopie. Les patients se plaignent fréquemment de douleur ipsilatérale à l'épaule après une vidéothoracoscopie; dans une étude, cette douleur a été rapportée comme étant chronique chez $10 \%$ des patients. ${ }^{5}$

La douleur postopératoire aiguë peut être provoquée par des processus myofasciaux ou neuropathiques produisant des changements neuroplastiques qui provoquent de la douleur chronique. Une sensibilisation périphérique et centrale peut survenir. L'objectif de l'analgésie préventive est, comme son nom l'indique, de prévenir la survenue de ces changements neuroplastiques et d'éviter ce qu'on appelle communément l'enroulement rachidien. La conclusion d'une méta-analyse portant sur 66 études de patients subissant divers types de chirurgie était que l'analgésie péridurale, l'infiltration de la plaie et l'administration systémique d'anti-inflammatoires non stéroïdiens (AINS) sont des techniques d'analgésie préventive efficaces, mais pas les opioïdes systémiques. ${ }^{6}$ Bien que la théorie soit séduisante, aucune étude n'a examiné l'efficacité de l'analgésie préventive pour traiter la douleur liée à une intervention par thoracoscopie. Des données prouvant son efficacité sont nécessaires, et tout particulièrement en ce qui touche à la douleur thoracoscopique postopératoire, avant que davantage de praticiens administrent une analgésie préventive.

Une péridurale installée au niveau $\mathrm{T} 4$ procure une analgésie très efficace pour traiter la douleur au site d'incision après une thoracotomie. Toutefois, les données démontrant que l'analgésie péridurale améliore le contrôle de la douleur après une thoracoscopie ne sont pas unanimement positives. En outre, les patients suivant des traitements anticoagulants ou antiplaquettaires pourraient ne pas être éligibles à recevoir une telle analgésie. Dans une étude randomisée chez des patients subissant une vidéothoracoscopie, on a observé que les patients recevant 
une analgésie péridurale montraient un contrôle de la douleur plus efficace durant le premier jour postopératoire que les patients témoins sans péridurale. ${ }^{7}$ Les patients $\mathrm{du}$ groupe témoin ont eu besoin d'analgésiques supplémentaires à des doses totales plus élevées que le groupe péridurale. Néanmoins, dans une étude précédente, l'analgésie péridurale thoracique n'a pas réduit l'incidence de douleur aiguë ou chronique après une pleurectomie par vidéothoracoscopie pour traiter un pneumothorax spontané primaire. ${ }^{8}$ L'injection unique de morphine intrathécale n'est pas fréquemment utilisée chez les patients subissant une vidéothoracoscopie et, à notre connaissance, il n'existe pas d'étude examinant cette modalité dans un contexte de vidéothoracoscopie.

La mise en place d'un bloc paravertébral (BPV) est une alternative appréciée à l'analgésie péridurale. Dans un récent sondage interrogeant les anesthésiologistes thoraciques du Royaume-Uni, on a découvert que l'utilisation de BPV avec une analgésie contrôlée par le patient (ACP) de morphine pour la lobectomie sous vidéothoracoscopie était bien plus courante $(50 \%)$ que le recours à une analgésie péridurale thoracique $(10 \%) .{ }^{9}$ Une injection d'anesthésique local au niveau paravertébral T4 peut provoquer un bloc ipsilatéral allant des dermatomes T2 à T6. Au contraire d'une anesthésie péridurale, un BPV ne provoque pas de sympathectomie, et l'hypotension est par conséquent moins fréquente. Un BPV peut être réalisé à l'aide d'une injection unique ou en installant un cathéter. Plusieurs données encourageantes ont été publiées concernant l'utilisation des BPV pour les vidéothoracoscopies. En 2005, Vogt et coll. ont démontré que les BPV amélioraient la douleur postopératoire chez les patients subissant une thoracoscopie jusqu'à $48 \mathrm{~h}$ après la chirurgie. ${ }^{10}$ Dans deux études subséquentes, il a été démontré que les BPV multiples et étagés réduisaient les scores de douleur et la toux, mais seulement durant les quatre et six premières heures suivant l'opération, respectivement. ${ }^{11,12}$ En raison de l'efficacité des $\mathrm{BPV}$, il n'est pas nécessaire de réaliser une péridurale pour les vidéothoracoscopies ayant peu de chances de se transformer en thoracotomie.

On peut également réaliser des blocs intercostaux, qui peuvent être effectués après le positionnement latéral du patient pour la chirurgie et sous anesthésie générale. Le risque de pneumothorax après un bloc intercostal est minimisé si ce dernier est réalisé pour une chirurgie thoracique, étant donné que le poumon opéré sera comprimé pendant la chirurgie, et qu'un drain thoracique sera de toute façon installé. Cette technique peut également être envisagée s'il existe une contre-indication à un bloc neuraxial, comme par exemple une coagulopathie. Dans les vidéothoracoscopies relativement mineures, telles que celles réalisées pour le drainage de liquide pleural ou une biopsie, l'intervention peut être réalisée à l'aide de blocs intercostaux et de sédation intraveineuse seulement. Dans de tels cas, le poumon ipsilatéral à la chirurgie s'affaissera pendant la respiration spontanée, et une technique d'anesthésie spéciale pour isoler le poumon ne sera pas nécessaire. Les blocs des nerfs intercostaux peuvent procurer aussi bien une anesthésie peropératoire qu'une analgésie postopératoire. Il a été rapporté que le recours à des blocs nerveux intercostaux réduisait la douleur et les besoins en morphiniques après une vidéothoracoscopie. ${ }^{13} \mathrm{Il}$ a également été rapporté que l'administration de bupivacaïne via un drain thoracique réduisait les scores de douleur et l'utilisation d'opioïdes en postopératoire après une vidéothoracoscopie.

L'analgésie multimodale réduit les besoins en opioïdes et leurs effets secondaires, y compris la dépression respiratoire, la nausée et le prurit. Les patients subissant une chirurgie thoracique ont en général des antécédents de tabagisme; en outre, ils pourraient souffrir d'une maladie pulmonaire obstructive chronique et ne pas tolérer les effets de dépression respiratoire des opioïdes systémiques. Bien que bon nombre d'anesthésiologistes, nous y compris, utilisent régulièrement des AINS en période postopératoire, il n'existe que très peu de données probantes spécifiques recommandant cette pratique chez les patients de chirurgie thoracoscopique. Dans un compte rendu, le traitement de la douleur postopératoire a été efficace chez une série consécutive de patients de vidéothoracoscopie à l'aide d'une perfusion de morphine sous-cutanée d'une durée maximale de deux jours et d'une administration régulière d'AINS. ${ }^{15}$ Certains chirurgiens pourraient préférer retarder l'administration de kétorolac jusqu'en postopératoire en raison d'inquiétudes quand aux effets inhibiteurs potentiels sur le système de coagulation. Le kétorolac peut être utile pour traiter les douleurs à l'épaule, qui ne seront probablement pas soulagées par une analgésie péridurale.

De meilleurs devenirs constitueraient une indication convaincante pour des techniques d'analgésie postopératoire spécifiques chez les patients de vidéothoracoscopie, mais les données à ce sujet sont rares. Dans une étude rétrospective, on a observé que moins de patients manifestaient une tachycardie supraventriculaire postopératoire après une thoracoscopie si une péridurale avait été installée au préalable. ${ }^{16}$ Une étude randomisée prospective subséquente a démontré une incidence réduite d'arythmies chez les patients bénéficiant d'une analgésie péridurale après une résection pulmonaire chez les patients atteints de cancer du poumon. ${ }^{17}$ Cette étude ne se limitait cependant pas à la thoracoscopie.

Selon nous, le plan d'analgésie postopératoire d'un patient de vidéothoracoscopie devrait commencer par une évaluation de l'ampleur de l'intervention, c'est-à-dire de la probabilité qu'elle se transforme en thoracotomie ouverte. Nombre de vidéothoracoscopies de courte durée 
ne nécessitent pas de péridurale, et on évite ainsi les risques qui y sont associés. Un patient devant subir une intervention d'ampleur limitée pourrait n'avoir besoin que de blocs intercostaux, de kétorolac et d'une ACP avec opioïdes pour traiter sa douleur postopératoire. Une péridurale ne serait pas requise. Sauf indication contraire, les AINS devraient également être utilisés.

Chez un patient devant subir une importante résection et pour lequel les risques de chirurgie ouverte sont élevés, il faudrait placer un cathéter péridural et une ACP activée immédiatement après la chirurgie. Il est possible que certains chirurgiens transforment régulièrement l'opération en thoracotomie si la biopsie initiale, réalisée à l'aide de la vidéothoracoscopie, est positive pour le cancer, et une péridurale devrait alors être installée d'office chez ces patients, sauf s'il y a contre-indication.

Selon nous, il existe de nombreuses occasions de recherche afin de découvrir les facteurs déterminant la technique à utiliser pour chaque vidéothoracoscopie. Dans notre pratique, la décision d'installer une péridurale thoracique chez un patient de vidéothoracoscopie dépend de la connaissance personnelle du chirurgien qu'a l'anesthésiologiste, de l'intervention, et du patient. Des études observationnelles et prospectives examinant les facteurs jouant un rôle dans le plan d'analgésie et les devenirs seraient très utiles.

Au-delà de la salle de réveil, une étape nécessaire dans nos recherches serait de mesurer les devenirs après vidéothoracoscopie et de les lier au plan d'analgésie périopératoire. Tout, de la satisfaction d'un patient à l'apparition de douleur chronique, en passant par les complications postopératoires, peut être lié au plan d'analgésie, mais nous n'avons aucune donnée appuyant ou réfutant cette hypothèse. Les recherches actuelles soutiennent que les techniques analgésiques postopératoires modernes améliorent la récupération des patients de vidéothoracoscopie, mais des analyses plus approfondies sont nécessaires.

Competing interests None declared.

\section{References}

1. Nagahiro I, Andou A, Aoe M, Sano Y, Date H, Shimizu N. Pulmonary function, postoperative pain, and serum cytokine level after lobectomy: a comparison of VATS and conventional procedure. Ann Thorac Surg 2001; 72: 362-5.
2. Steegers MA, Snik DM, Verhagen AF, van der Drift MA, WilderSmith $\mathrm{OH}$. Only half of the chronic pain after thoracic surgery shows a neuropathic component. J Pain 2008; 9: 955-61.

3. Furrer M, Rechsteiner R, Eigenmann $V$, Signer $C$, Althaus $U$, Ris $H B$. Thoracotomy and thoracoscopy: postoperative pulmonary function, pain and chest wall complaints. Eur J Cardiothorac Surg 1997; 12: 82-7.

4. Wildgaard K, Ravn J, Kehlet $H$. Chronic post-thoracotomy pain: a critical review of pathogenic mechanisms and strategies for prevention. Eur J Cardiothorac Surg 2009; 36: 170-80.

5. Passlick B, Born $C$, Sienel W, Thetter $O$. Incidence of chronic pain after minimal-invasive surgery for spontaneous pneumothorax. Eur J Cardiothorac Surg 2001; 19: 355-8.

6. Ong CK, Lirk P, Seymour RA, Jenkins BJ. The efficacy of preemptive analgesia for acute postoperative pain management: a meta-analysis. Anesth Analg 2005; 100: 757-73.

7. Yoshioka M, Mori T, Kobayashi H, et al. The efficacy of epidural analgesia after video-assisted thoracoscopic surgery: a randomized control study. Ann Thorac Cardiovasc Surg 2006; 12: 313-8.

8. Fernandez MI, Martin-Ucar AE, Lee HD, West KJ, Wyatt R, Waller DA. Does a thoracic epidural confer any additional benefit following video-assisted thoracoscopic pleurectomy for primary spontaneous pneumothorax? Eur J Cardiothorac Surg 2005; 27: 671-4.

9. Kotemane NC, Gopinath N, Vaja R. Analgesic techniques following thoracic surgery: a survey of United Kingdom practice. Eur J Anaesthesiol 2010; 27: 897-9.

10. Vogt A, Stieger DS, Theurillat C, Curatolo M. Single-injection thoracic paravertebral block for postoperative pain treatment after thoracoscopic surgery. Br J Anaesth 2005; 95: 816-21.

11. Kaya FN, Turker G, Basagan-Mogol E, Goren S, Bayram S, Gebitekin $C$. Preoperative multiple-injection thoracic paravertebral blocks reduce postoperative pain and analgesic requirements after video-assisted thoracic surgery. J Cardiothorac Vasc Anesth 2006; 20: 639-43.

12. Hill SE, Keller RA, Stafford-Smith M, et al. Efficacy of singledose, multilevel paravertebral nerve blockade for analgesia after thoracoscopic procedures. Anesthesiology 2006; 104: 1047-53.

13. Bolotin $G$, Lazarovici H, Uretzky G, Zlotnick AY, Tamir A, Saute $M$. The efficacy of intraoperative internal intercostal nerve block during video-assisted thoracic surgery on postoperative pain. Ann Thorac Surg 2000; 70: 1872-5.

14. Demmy TL, Nwogu C, Solan P, Yendamuri S, Wilding G, DeLeon O. Chest tube-delivered bupivacaine improves pain and decreases opioid use after thoracoscopy. Ann Thorac Surg 2009; 87: 1040-6.

15. Kamiyoshihara M, Nagashima T, Ibe T, Atsumi J, Shimizu K, Takeyoshi $I$. Is epidural analgesia necessary after video-assisted thoracoscopic lobectomy? Asian Cardiovasc Thorac Ann 2010; 18: 464-8.

16. Neustein SM, Kahn P, Krellenstein D, Cohen E. Incidence of arrhythmias after thoracic surgery: thoracotomy versus videoassisted thoracoscopy. J Cardiothorac Vasc Anesth 1998; 12: 659-61.

17. Oka T, Ozawa T, Ohkubo Y. Thoracic epidural bupivacaine attenuates supraventricular tachyarrhythmias after pulmonary resection. Anesth Analg 2001; 93: 253-9. 